\title{
Protective Control for Robot Manipulator by Sliding Mode Based Disturbance Reconstruction Approach
}

\author{
Yiyong Sun ${ }^{1,2}$, Zengjie Zhang ${ }^{2}$, Marion Leibold ${ }^{2}$, Rameez Hayat ${ }^{2}$, Dirk Wollherr ${ }^{2}$ and Martin Buss ${ }^{2}$
}

\begin{abstract}
This paper presents a protective control framework for robot manipulators using sliding mode based state estimation and disturbance reconstruction. Specifically, the nonlinear dynamic state-space model of the robot is transformed into a descriptor form, allowing the design of a sliding mode observer and a sliding mode based trajectory tracking control. Different reaction strategies to protect the robot manipulator are presented according to the strength of the disturbance and whether the environment is completely perceptible. Finally, to show the effectiveness of this novel combination of sliding mode observer and protective controller, an experiment on a two degree-of-freedom manipulator is conducted.
\end{abstract}

\section{INTRODUCTION}

With the increasing demand of interaction and cooperation between humans and robots, the safety of human collaborators and the self-protectability of the robots has become a critical issue in human-robot interaction (HRI) related tasks. This includes how to handle unexpected faults of robot components and collisions between robots and the environment, even humans. Even though different trajectory schemes based on collision avoidance have been proposed in the past decades [1], collisions may also happen due to trajectory deviations or unmodelled environment dynamics. For years, isolating robots from humans solved this problem [2]. While nowadays, external sensors such as proximity sensors [3], image sensors [4], [5], strain gauges [6] and sensitive skins [7] are utilized to detect manipulator collisions and system faults, such that the safety of HRI is guaranteed.

Even though external sensors proved to be powerful in detecting collisions and faults, they bring up cost and involve reliability issues to the system. It has become popular to utilize system 'analytic redundancy' to induce an effective 'residual signal' by which the profile of collision impact can be indicated [8], which contributes to the disturbance estimation problem. It has been suggested that the internal impacts, e.g. joint actuator faults and the external impacts, e.g. collision with the environment, of robots can be modelled as disturbance torques exerted on robot joints, such that both problems can be formulated as disturbance estimation issues and solved under the framework of fault detection and isolation (FDI) [9]. In this paper we extend the concept of "collision" to also cover the contact with pulling forces. Thus the profile of the internal or external torques can be reconstructed using a disturbance observer with the information only from the measurement of joint encoders, after which

\footnotetext{
${ }^{1}$ Research Institute of Intelligent Control and System, Harbin Institute of Technology, 150001, Harbin, China.

2 Chair of Automatic Control Engineering, Technical University of Munich, 80333, Munich, Germany
}

a protective reaction controller can be conducted to protect the manipulator and human cooperators in the environment from further impact.

A number of approaches have been proposed to solve disturbance estimation problems in HRI scenarios [2], [10], [11], [12]. In [2], the collision force is calculated using the desired trajectory and the commanded torques on the joints. This approach only performs well when the current trajectory of the robot consists with the desired trajectory, which does not always happen. In [10], [11] the generalized momentum method is introduced to compute the disturbance. The approach is efficient in terms of computational cost since it does not require the inverse of the inertia matrix, but joint velocities are required which are obtained after being filtered from the derivative of joint positions. Among those popular methods, sliding mode theory stands out for its advantage of insensitivity and adaptivity towards disturbance and model uncertainties. Firstly applied on linear systems [13], [14], [15] and later extended to nonlinear systems [16], [17], [18], sliding mode observer (SMO) approaches have recently been used for disturbance estimation (DE) and fault diagnosis and isolation (FDI) of robot manipulators [19], [20]. However, in these approaches, either the measurement of both joint angular positions and joint velocities is available, or the disturbance on different joints are not coupled with the timevarying parameters. However in many practical cases, the joint velocities are calculated by taking the derivative of position measurements, which is contaminated by noise. Thus derivative-based velocity acquisition is not an appropriate solution for disturbance observation.

Inspired by methods in [13], [21], [22], we design a SMO that estimates robot joint velocities and disturbance torques, as well as a sliding mode controller (SMC) that governs the trajectory tracking and the reaction strategy after collision or system fault. In this approach, no external sensors are needed for disturbance handling. The reconstructed disturbance torques are then used for the design of the protective controller. Specifically, when the disturbance is weak, the disturbance is supressed by the controller such that the desired trajectory is tracked; while for strong disturbance, proper protective reaction is taken to protected from further impact [23].

By contributing to the combination of a sliding mode disturbance observer and a sliding mode controller for robot manipulators, we propose a complete framework for robot disturbance observation and reaction strategy, which can be widely applied to critical topics such as collision detection and reaction, as well as fault detection and reaction. Our 
scheme has been tested and successfully applied on a twodegree-of-freedom (DoF) manipulator system as shown in Fig. 1, which will be discussed in details later.

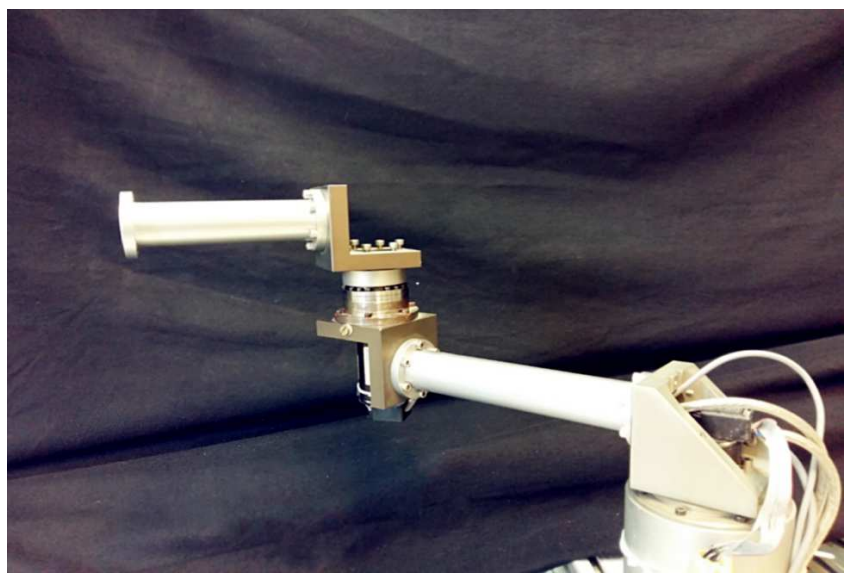

Fig. 1: The 2-DoF Robot Manipulator for the Experiment

The rest of this paper is organized as follows. The assumptions and the state-space dynamic model of the robot control system are given in Section II. Section III presents a system transformation, the observer design, the error system analysis, the trajectory tracking controller, reaction strategies and the design procedures. An experiment is provided in Section IV to examine the validity of the scheme in robot control. Section V concludes the paper.

\section{PRELIMINARIES}

Based on the Euler-Lagrange equations [24], the dynamic model of an $n$-joint robot manipulator can be written as

$$
\tau=M(q) \ddot{q}+C(q, \dot{q}) \dot{q}+G(q)+F(\dot{q}),
$$

where $M(q) \in \mathbb{R}^{n \times n}, C(q, \dot{q}) \in \mathbb{R}^{n \times n}, G(q) \in \mathbb{R}^{n \times 1}$ and $F(\dot{q}) \in$ $\mathbb{R}^{n \times 1}$ are inertia matrix, coriolis and centrifugal matrix, gravitational and frictional vectors of the manipulator. $q(t) \in \mathbb{R}^{n \times 1}$ is the vector of joint angles, while $\tau(t) \in \mathbb{R}^{n \times 1}$ is the vector of torques applied on robot joints. To be brief, in the following, we omit the explicit ' $t$ ' for the variables that depend on time. Note that the joint torque $\tau$ is the combination of the commanded torque vector $u$ and the disturbance torque $\tau_{d}$ :

$$
\tau=u+\tau_{d}
$$

Considering that only the joint angle $q$ is measurable, (1) can be further rewritten as

$$
\left\{\begin{array}{l}
\dot{x}=A x+B g(x, u)+B M^{-1}(\Upsilon x) \tau_{d}, \\
y=C x
\end{array}\right.
$$

where $x=\left[\begin{array}{ll}q^{T} & \dot{q}^{T}\end{array}\right]^{T} \in \mathbb{R}^{2 n \times 1}$ is the state and $y \in \mathbb{R}^{n \times 1}$ is the measurable output, and

$$
\begin{aligned}
& A=\left[\begin{array}{cc}
0 & I_{n} \\
0 & 0
\end{array}\right], \quad B=\left[\begin{array}{c}
0 \\
I_{n}
\end{array}\right], \quad C=\left[\begin{array}{cc}
I_{n} & 0
\end{array}\right], \\
& g(x, u)=-M^{-1}(\Upsilon x)[C(\Upsilon x, \dot{q}) \dot{q}+G(q)+F \dot{q}-u], \\
& q=\Upsilon x, \quad \text { and } \Upsilon=\left[\begin{array}{ll}
I_{n} & 0
\end{array}\right]
\end{aligned}
$$

are system parameter matrices, and $I_{n} \in \mathbb{R}^{n \times n}$ is the $n$ dimensional identity matrix. In order to apply our method, we make the following assumptions on the non-linear term in (3) and the constraints for the disturbance torque $\tau_{d}$.

Assumption 1: The nonlinear function $g(x, u)$ satisfies the Lipschitz condition, i.e. with any $x_{1}, x_{2} \in \mathbb{R}^{2 n \times 1}$, there exists $\gamma \geq 0$, such that [25], [26]

$$
\left\|g\left(x_{2}, u\right)-g\left(x_{1}, u\right)\right\| \leq \gamma\left\|x_{2}-x_{1}\right\| .
$$

Assumption 2: The torque disturbance $\tau_{d}$ is bounded by

$$
\left\|\tau_{d}\right\| \leq \tau_{M},\left\|\dot{\tau}_{d}\right\| \leq \tau_{m}
$$

where $\tau_{M}, \tau_{m}>0$ are boundary values that should be determined by experiments. For simplification, we define an equivalent decoupled disturbance as

$$
f_{d}=M^{-1}(\Upsilon x) \tau_{d}
$$

According to Assumption 2, this decoupled disturbance is also bounded by

$$
\left\|f_{d}\right\| \leq f_{M},\left\|\dot{f}_{d}\right\| \leq f_{m}
$$

where $f_{M}, f_{m}>0$ are positive boundary values which can be determined in experiments. Thus (3) can be rewritten as

$$
\left\{\begin{array}{l}
\dot{x}=A x+B g(x, u)+B_{f} f_{d}, \\
y=C x
\end{array}\right.
$$

where $B_{f}=\left[\begin{array}{ll}0 & I_{n}\end{array}\right]^{T}$. Similar to the method in [22], [27], we transform (8) into a descriptor form to enable the estimation of the full state and the disturbance $f_{d}$ by introducing the following auxiliary differential equation

$$
\dot{f}_{d}=\Phi f_{d}-\Phi f_{d}+\dot{f}_{d}
$$

with an auxiliary disturbance variable being defined as

$$
\bar{f}=f_{d}+\Phi^{-1} \dot{f}_{d}
$$

where $\Phi>0$ is a properly selected scaling matrix. Thus by constructing a new augmented state vector $\bar{x}=\left[\begin{array}{cc}x^{T} & f_{d}^{T}\end{array}\right]^{T}$ the dynamic equation of the system can be written as

$$
\left\{\begin{aligned}
\bar{E} \dot{\bar{x}} & =\bar{A} \bar{x}+\bar{B} g(x, u)+\bar{B}_{f} \bar{f}, \\
y & =\bar{C} \bar{x}
\end{aligned}\right.
$$

where

$$
\begin{aligned}
& \bar{E}=\left[\begin{array}{cc}
I_{2 n} & B_{f} \Phi^{-1} \\
0 & I_{n}
\end{array}\right], \quad \bar{A}=\left[\begin{array}{cc}
A & 0 \\
0 & -\Phi
\end{array}\right], \\
& \bar{B}=\left[\begin{array}{c}
B \\
0
\end{array}\right], \quad \bar{B}_{f}=\left[\begin{array}{c}
B_{f} \\
\Phi
\end{array}\right], \quad \bar{C}=\left[\begin{array}{ll}
C & 0
\end{array}\right] .
\end{aligned}
$$

Our target in this paper is to design a control strategy based on the full estimation of the system state $\hat{x}$ and the reconstruction of disturbance $\hat{\tau}_{d}$, so that the protective mechanism of the manipulator can react on disturbance appropriately in various situations. 


\section{Disturbance OBSERVER AND CONTROLLER DESIGN}

\section{A. SMO design}

For the estimation of the unknown state and disturbance in $\bar{x}$, the following sliding mode observer is designed:

$$
\left\{\begin{aligned}
\bar{E} \dot{\bar{x}} & =\bar{A} \hat{\bar{x}}+\bar{B} g(\hat{x}, u)+\bar{L}_{f} u_{s}+\bar{L}(y-\bar{C} \hat{\bar{x}}), \\
\hat{y} & =\bar{C} \hat{\bar{x}}
\end{aligned}\right.
$$

where $\hat{\bar{x}}=\left[\begin{array}{cc}\hat{x}^{T} & \hat{f}_{d}^{T}\end{array}\right]^{T}$ is the estimation of the augmented state $\bar{x}$, and $\bar{L}, \bar{L}_{f}$ are properly chosen gain matrices while $u_{s}$ is the discontinuous input to be designed.

Combining the SMO (12) and the descriptor model (11), the error dynamics can be obtained as

$$
\bar{E} \dot{\bar{e}}=(\bar{A}-\bar{L} \bar{C}) \bar{e}+\bar{B} \bar{e}_{g}(\hat{x}, x, u)+\bar{L}_{f} u_{s}-\bar{B}_{f} \bar{f},
$$

where $\bar{e}=\hat{\bar{x}}-\bar{x}$ and $\bar{e}_{g}(\hat{x}, x, u)=g(\hat{x}, u)-g(x, u)$. According to Assumption 1, a positive parameter $\gamma$ exists such that $\left\|\bar{e}_{g}(\hat{x}, x, u)\right\|=\|g(\hat{x}, u)-g(x, u)\| \leq \gamma\|\hat{x}-x\|$.

With the discontinuous input gain matrix defined as $\bar{L}_{f}=$ $\bar{B}_{f}$, the discontinuous input $u_{S}$ in (12) is chosen as

$$
u_{s}=-\left(f_{M}+\max \left|\lambda\left(\Phi^{-1}\right)\right| f_{m}+\zeta\right) \operatorname{sgn}(s),
$$

where the constant $\zeta>0$ can be chosen as $\zeta \ll f_{M}+$ $\max \left|\lambda\left(\Phi^{-1}\right)\right| f_{m}$ and $\Phi$ should be selected such that the amplitude of the constant gain $\left(f_{M}+\max \left|\lambda\left(\Phi^{-1}\right)\right| f_{m}\right)$ in $u_{s}$ is small. The switching function $s$ is selected as

$$
s=H \bar{C} \bar{e},
$$

where $H \in \mathbb{R}^{n \times n}$ is defined by the bounded constraint

$$
(H \bar{C})^{T}=\bar{P} \bar{E}^{-1} \bar{B}_{f}
$$

where $\bar{P}$ is the Lyapunov matrix of the error system (13). The LMI-based method to solve 12 and to find a proper gain matrix $H$ is presented in Appendix I.

A critical issue to guarantee the stability of the error dynamics of the SMO is the appropriate choice of a gain matrix $\bar{L}$. In [21], together with the stability analysis of observation error dynamics, the observer gain is selected as $\bar{L}=\bar{E} \bar{Q}^{-1} \bar{C}^{T}$. While following Lemma 2 in [28] and Lemma 3 in [27], we design the high gain $\bar{L}$ for the SMO as in Lemma 1, which proves to be more successful and effective during experimental verification.

Lemma 1: If the descriptor system (11) is observable, the matrix $\bar{E}^{-1}(\bar{A}-\bar{L} \bar{C})$ can be designed to be Hurwitz with an appropriate gain $\bar{L}$.

Proof: See Appendix II.

The sufficient condition for the stability of the error system (13) is shown in Theorem 1.

Theorem 1: With the designed observer gain $\bar{L}$ determined by Lemma 1 , the discontinuous function $u_{s}$ and its gain matrix $L_{f}$ defined above, if there exists a positive constant $\gamma \geq 0$ and matrices $\bar{P} \geq 0$ and $H$ with appropriate dimensions, such that the bounded constraint (16) and the following constraint are satisfied:

$\left(\bar{P} \bar{E}^{-1}(\bar{A}-\bar{L} \bar{C})+\gamma \bar{P} \bar{B} T_{e}\right)+\left(\bar{P} \bar{E}^{-1}(\bar{A}-\bar{L} \bar{C})+\gamma \bar{P} \bar{B} T_{e}\right)^{T}<0$, then the error system (13) is asymptotically stable.

Proof: See Appendix III.

It is worth mentioning that we thus extend the methods of SMO design from linear systems [27], [28] to the nonlinear case (11).

With the observer shown in (12), the reconstructed values of system state $\hat{x}=\left[\begin{array}{cc}\hat{q}^{T} & \dot{\hat{q}}^{T}\end{array}\right]^{T}$, and the disturbance torque

$$
\hat{\tau}_{d}=M(\Upsilon x) \hat{f}_{d}
$$

is obtained.

\section{B. Sliding mode based trajectory tracking controller}

If the reconstructed disturbance $\hat{\tau}_{d}$ is weak, which means there is assumed to be no collision, the manipulator is supposed to be able to track the desired trajectory $x_{d}=$ $\left[\begin{array}{cc}q_{d}^{T} & \dot{q}_{d}^{T}\end{array}\right]^{T}$, where $q_{d}, \dot{q}_{d} \in \mathbb{R}^{n}$ are respectively desired joint angular positions and velocities of the robot. Considering the system dynamics (3), the nonlinear term $B g(x, u)$ is treated as fictitious input $\mathscr{U}$, and the real control input $u$ is calculated as

$$
u=g^{-1}\left(x, B^{+} \mathscr{U}\right)
$$

where $B^{+}$is the pseudo-inverse of $B$ defined as

$$
B^{+}=\left(B^{T} B\right)^{-1} B^{T} \text {. }
$$

Thus we can rewrite (8) as

$$
\left\{\begin{array}{l}
\dot{x}=A x+\mathscr{U}+B_{f} f_{d}, \\
y=C x .
\end{array}\right.
$$

With the SMO (12), we obtain

$$
\dot{\hat{x}}=A \hat{x}+\hat{\mathscr{U}}+L_{f 1} u_{s}+\bar{L}_{1}(y-C \hat{x})-B_{f} \Phi^{-1} \dot{\hat{f}}_{d},
$$

with $L_{f 1}=B_{f}$ and $\hat{\mathscr{U}}=B g(\hat{x}, u)$. Additionally with the auxiliary equation $\dot{x}_{d}=A x_{d}-A x_{d}+\dot{x}_{d}$, and the tracking error $\varsigma=x_{d}-\hat{x}$, we obtain

$$
\dot{\varsigma}=A\left(\varsigma-x_{d}\right)+\dot{x}_{d}-\hat{\mathscr{U}}-\bar{L}_{1}(y-C \hat{x})+B_{f}\left(\Phi^{-1} \dot{\hat{f}}_{d}-u_{s}\right) .
$$

A critical issue here is to choose a proper $\hat{\mathscr{U}}$ in order to obtain a stable tracking error dynamics (23) in the form $\dot{\zeta}=\mathscr{H} \varsigma$ with $\mathscr{H}$ being Hurwitz. Before introducing the controller, we choose the switching function as

$$
\hat{s}=G \varsigma-G \int_{0}^{t}(A+K) \varsigma(\delta) d \delta .
$$

where the parameter matrix $G$ is positive definite, $G>0$. So the derivative of the switching function $\hat{s}$ is

$$
\dot{\hat{s}}=G\left[-A x_{d}+\dot{x}_{d}-\hat{\mathscr{U}}-B_{f} u_{s}-\bar{L}_{1}(y-C \hat{x})\right]+B_{f} \Phi^{-1} \dot{\hat{f}}_{d}-K \varsigma .
$$

By setting $\dot{\hat{s}}=0$, we have

$$
\hat{\mathscr{U}}_{e q}=-A x_{d}+\dot{x}_{d}-B_{f} u_{s}-\bar{L}_{1}(y-C \hat{x})+B_{f} \Phi^{-1} \dot{\hat{f}}_{d}-K \varsigma .
$$

Substituting (26) into (23), it follows that

$$
\dot{\zeta}=(A+K) \varsigma \text {. }
$$


It can be seen that, if $A+K$ is Hurwitz, the tracking error dynamics (27) is stable. The gain $K$ is selected by pole placement. The element $\dot{\hat{f}}_{d}$ and the discontinuous function $u_{s}$ cannot be introduced into the control input for the reason that $\dot{\hat{f}}_{d}$ is not available and $u_{s}$ can cause more chattering. Therefore, we redesign the control input as

$$
u=M(\hat{q}) B^{+} \hat{\mathscr{U}}_{\kappa}+C(\hat{q}, \dot{\hat{q}}) \dot{\hat{q}}+G(\hat{q})+F \dot{\hat{q}},
$$

where

$$
\hat{\mathscr{U}}_{\kappa}=-A x_{d}+\dot{x}_{d}-\bar{L}_{1}(y-C \hat{x})-K \varsigma+\hat{\mathscr{U}}_{s} .
$$

The discontinuous element $\hat{\mathscr{U}}_{s}$ is designed to replace $B_{f} \Phi^{-1} \dot{\hat{f}}_{d}$ and $B_{f} u_{s}$

$$
\hat{\mathscr{U}}_{s}=\left(-\left\|B_{f} \Phi^{-1} f_{m}\right\|-\left\|B_{f} f_{M}+B_{f} \Phi^{-1} f_{m}\right\|-\vartheta\right) \operatorname{sgn}(\hat{s}),
$$

where $\vartheta \geq 0$. With the control input (28) and (29), we analyze the reachability of the controller as in the following theorem.

Theorem 2: With the SMO in (12) and the controller in (28), the error $\varsigma$ approaches the surface (24) in finite time and remains there in subsequent time.

Proof: We define the Lyapunov function

$$
V=0.5 \hat{s}^{T} \hat{s} \text {. }
$$

Using (25) and (28), we obtain the derivative of $V$ as

$$
\begin{aligned}
\dot{V} & =\hat{s}^{T} \dot{\hat{s}} \\
& =\hat{s}^{T} G\left(-\hat{\mathscr{U}}_{s}-B_{f} u_{s}+B_{f} \Phi^{-1} \dot{\hat{f}}_{d}\right) .
\end{aligned}
$$

Thus, with $G \geq 0$ and (30), it follows that

$$
\dot{V} \leq \hat{s}^{T} G(-\vartheta \operatorname{sgn}(\hat{s})) \leq 0 .
$$

Hereupon, if (32) holds, the reachability of the switching function is proved.

\section{Protective reaction strategy}

When the observed disturbance is strong, it indicates that a collision force acts on the robot. In this case, the robot should not be forced to track the desired trajectory discarding the disturbance force, otherwise the strong contact force might cause unrecoverable damages to the environment. Therefore we suggest that a protective reaction strategy should be taken when a strong disturbance is detected, such that the desired trajectory of the robot can be modified and safety of both robot and the environment can be guaranteed. Hence, we introduce the following two protective strategies considering different situations:

1) If the position of the obstacle is not known, the desired trajectory is modified. Instead, the control input $u$ of the robot in terms of (28) is bounded;

2) If the position of the obstacle is known, the desired trajectory $x_{d}$ of the robot is changed accordingly, and the control input $u$ should also be bounded.

Fig. 2 shows the geometrical relation between a $n$-DoF manipulator and the colliding obstacle before a collision happens. Assuming anti-clockwise to be the positive direction,

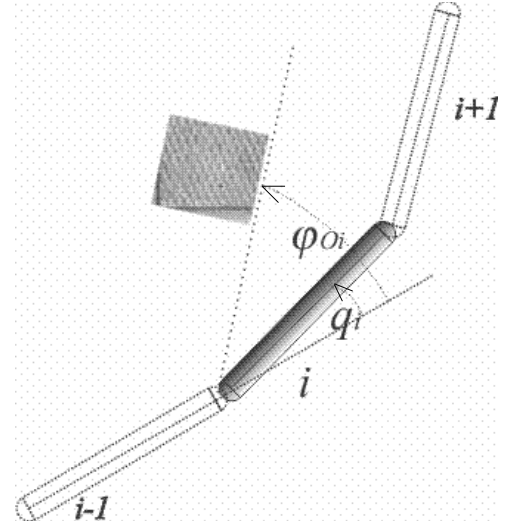

Fig. 2: Disturbance on $i$-th joint

the angular distance between the obstacle and the $i$-th link can be defined as

$$
\varphi_{p, i}=\varphi_{O, i}-q_{i},
$$

where $\varphi_{O, i}, q_{i} \in(-\pi, \pi)$ are respectively the positions of the obstacle and link $i$ in the joint space coordinate, and $i=$ $1, \cdots, n$. Thus the obstacle distance vector $\varphi_{P} \in \mathbb{R}^{n \times 1}$ can be defined as

$$
\varphi_{p}=\left[\begin{array}{lll}
\varphi_{p, 1}^{T} & \ldots & \varphi_{p, n}^{T}
\end{array}\right]^{T} .
$$

Under the definition given above, the protective strategies can be specifically described as follows.

Strategy 1 : When the value of $\varphi_{P}$ is not available, we have no information about the position of the obstacle. In this case, we define a saturated control input $\tilde{u} \in \mathbb{R}^{n \times 1}$ in place of the original input $u$ :

$$
\tilde{u}_{i}= \begin{cases}-u_{\delta, i}, & u_{i} \in\left(-\infty,-u_{\delta, i}\right], \\ u_{i}, & u_{i} \in\left(-u_{\delta, i}, u_{\delta, i}\right), \\ u_{\delta, i}, & u_{i} \in\left[u_{\delta, i},+\infty\right),\end{cases}
$$

where $u_{i}$ is the $i$-th element of $u$, and $u_{\delta, i}$ is the positive saturation value of control input defined as

$$
u_{\delta, i}=\delta_{i} e^{-\left|k_{i} \hat{\tau}_{d, i}\right|},
$$

and $\delta_{i}, k_{i}>0$ are positive parameters that are determined in the experiment. $\hat{\tau}_{d, i}$ is the reconstructed disturbance torque of $i$-th joint of the robot.

Strategy 2 : If the value of $\varphi_{P}$ is available, we have the information about the position of the obstacle. In this case, the desired trajectory of the robot is changed, such that the possible damage is reduced. Here we define the modified trajectory as

$$
x_{d, c}=\left[\begin{array}{ll}
\tilde{q}_{d}^{T} & \dot{\tilde{q}}_{d}^{T}
\end{array}\right]^{T},
$$

where

$$
\tilde{q}_{d}=q+\int_{t}^{t+\Delta t} \dot{\tilde{q}}_{d}^{T} d t
$$

and

$$
\dot{\tilde{q}}_{d, i}= \begin{cases}l_{s, i} \dot{\hat{q}}_{i} e^{k_{s, i}}\left(\left|\hat{\tau}_{d, i}\right|-\tau_{s, i}\right), & \mid \begin{array}{l}
\hat{\tau}_{d, i} \mid-\tau_{s, i}>0, \\
\dot{q}_{d},
\end{array} \\
\hat{\tau}_{d, i} \mid-\tau_{s, i} \leqslant 0,\end{cases}
$$


where $t$ is the current time, $\Delta t$ is an adjustable interval and $l_{s, i}$ and $k_{s, i}$ are scaling factors. The basic idea behind this strategy is to take advantage of the prior knowledge about the environment to modify the desired trajectory such that the contact force between the robot and the obstacle vanishes. In this paper we propose two practical scenarios to demonstrate the protective strategies. The first one is a ball hitting on the robot causing a collision; while the second one is a rope accidentally trapping the robot preventing it from moving forward. It is worth mentioning that the first case has been considered by previous research [2]. The second scenario, however, is still rarely studied. By proposing our protective control framework, we extend collision reaction to a broader range such that previous work is generalized. For the two cases mentioned above, the parameters involved in (39) can be determined according to Tab. I.

TABLE I: Reaction strategies in different situations

\begin{tabular}{|c|c|c|c|c|c|}
\hline \hline$\phi_{p, i}$ & $\hat{\tau}_{d, i}$ & Type & $\dot{q}_{i}$ & $l_{s, i}$ & $k_{s, i}$ \\
\hline & + & \multirow{2}{*}{ ball pushing } & + & 1 & $+\left|k_{s, i}\right|$ \\
+ & + & & - & -1 & $-\left|k_{s, i}\right|$ \\
& - & \multirow{2}{*}{ rope pulling } & + & -1 & $-\left|k_{s, i}\right|$ \\
& - & & - & 1 & $+\left|k_{s, i}\right|$ \\
\hline & + & \multirow{2}{*}{ rope pulling } & + & 1 & $+\left|k_{s, i}\right|$ \\
- & + & & -1 & $-\left|k_{s, i}\right|$ \\
& - & \multirow{2}{*}{ ball pushing } & + & -1 & $-\left|k_{s, i}\right|$ \\
& - & & - & 1 & $+\left|k_{s, i}\right|$ \\
\hline \hline
\end{tabular}

Remark 1: When $\dot{\hat{q}}_{i}=0$, the commanded torque input follows strategy I. Thus a combination of strategy 1 and strategy 2 is possible and straightforward.

Remark 2: The control strategy designed in this paper can protect the robot given the dynamic equations of motion and information on the current configuration of the manipulator. The method is based on the improvement of the 5th reaction strategy in [2], we generalized the collision cases to a broader range of interaction of robot and the environment.

\section{EXPERIMENT}

In this section, an experiment is conducted to evaluate the protective reaction strategies. The sampling time in this experiment is set to $1 \mathrm{~ms}$. The 2-DoF manipulator used for experimental validation is shown in Fig. 1, whose parameter matrices are

$$
\begin{aligned}
M(q) & =\left[\begin{array}{cc}
0.442+0.0286 \cos \left(q_{2}\right) & * \\
0.0088+0.0143 \cos \left(q_{2}\right) & 0.2226
\end{array}\right], \\
C(q, \dot{q}) & =\left[\begin{array}{cc}
-0.029 \sin \left(q_{2}\right) \dot{q}_{2} & -0.014 \sin \left(q_{2}\right) \dot{q}_{2} \\
0.014 \sin \left(q_{2}\right) \dot{q}_{1} & 0
\end{array}\right], F(\dot{q})=
\end{aligned}
$$

where $c_{1}, c_{2}=2.6 \times 10^{-4}$ are frictional coefficients of the joints. Gravity impact is ignored due to the horizontal construction. The matrices $A, B$ and $C$ are chosen as in (3). The initial values of $x, \hat{x}, \tau_{d}$ and $\hat{\tau}_{d}$ are all set to zero. According to the approach presented in Section III, a SMO and a SMC are designed before testing the reaction strategies.

(i) Set the parameters matrices as $\Phi=-36 I_{n}, \bar{L}_{f}=\bar{B}_{f}$ and $\kappa=0.1$. The gain $\bar{L}$ is chosen as

$$
\bar{L}=\left[\begin{array}{cccccc}
144.6 & 0 & 5241.8 & 0 & 262.1 & 0 \\
0 & 144.6 & 0 & 5241.8 & 0 & 262.1
\end{array}\right] \text {. }
$$
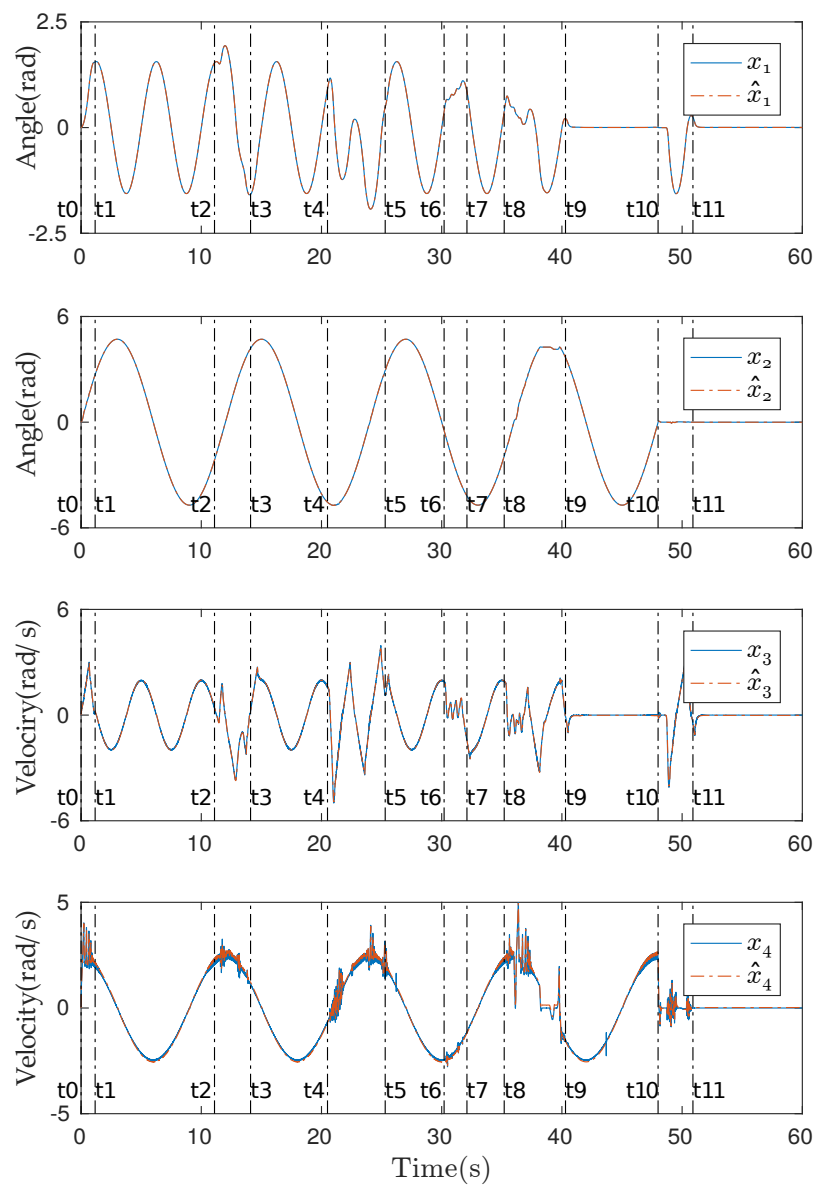

Fig. 3: State $x$ and its estimation $\hat{x}$
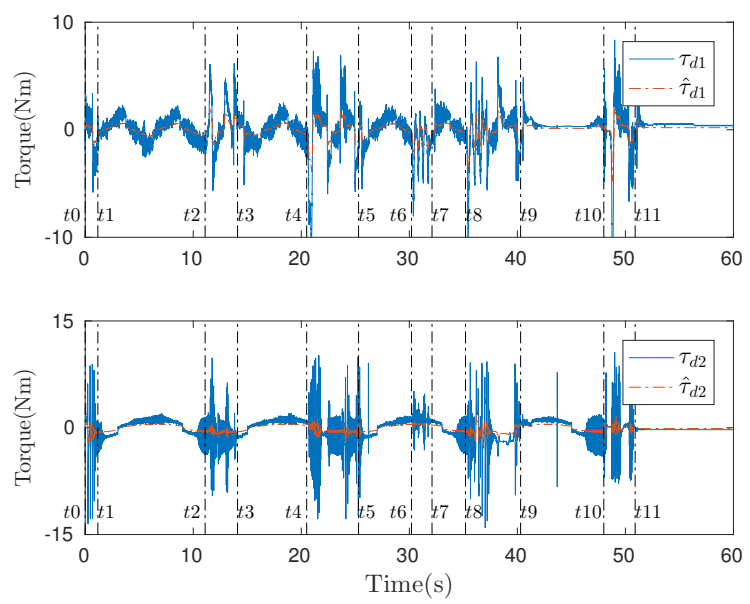

Fig. 4: Disturbance $\tau_{d}$ and its estimation $\hat{\tau}_{d}$ 


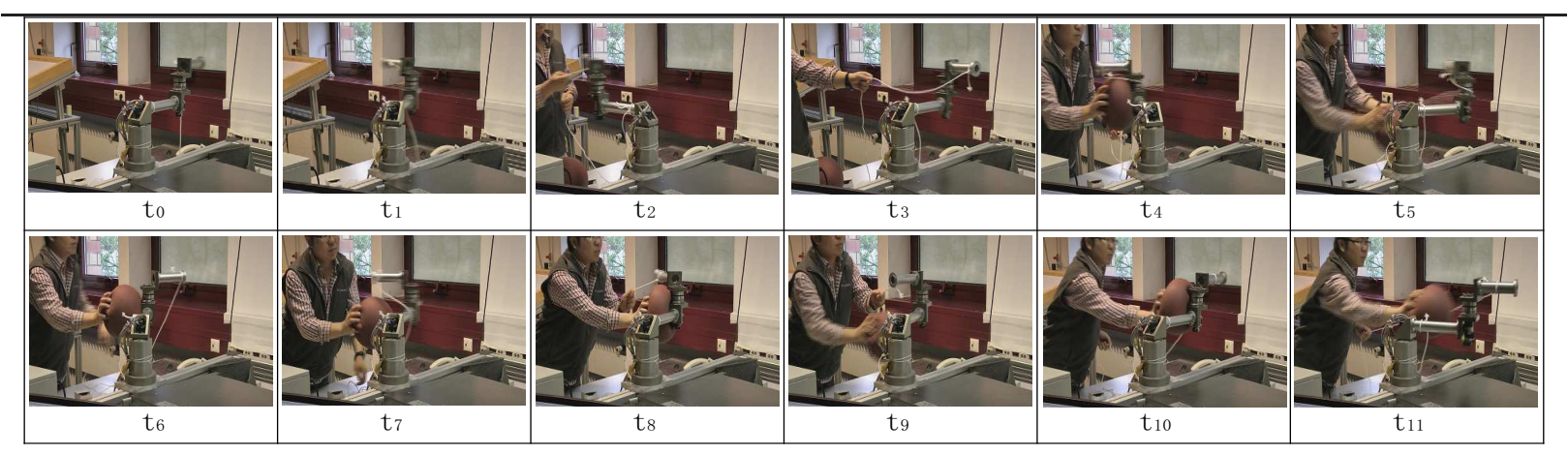

TABLE II: Disturbances exerted by human
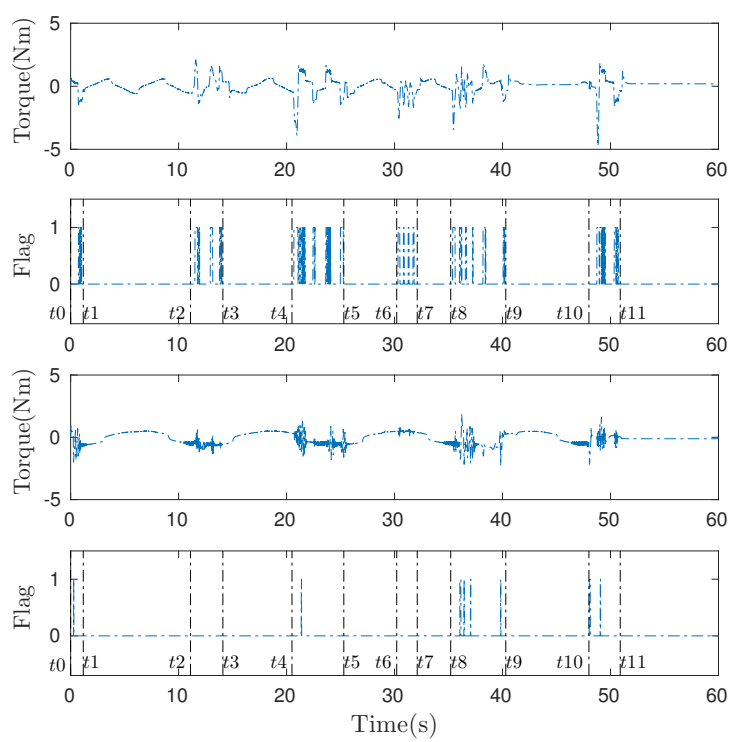

Fig. 5: The reconstructed disturbance $\hat{\tau}_{d}(1$ and 3$)$ and the corresponding flag signals (2 and 4) showing whether the disturbance is strong or weak.
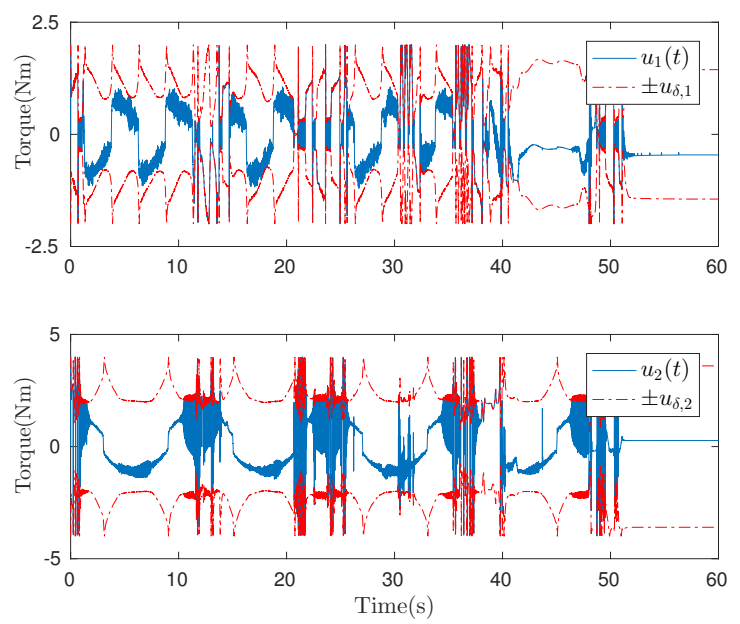

Fig. 6: Control input $u$ and its bounds
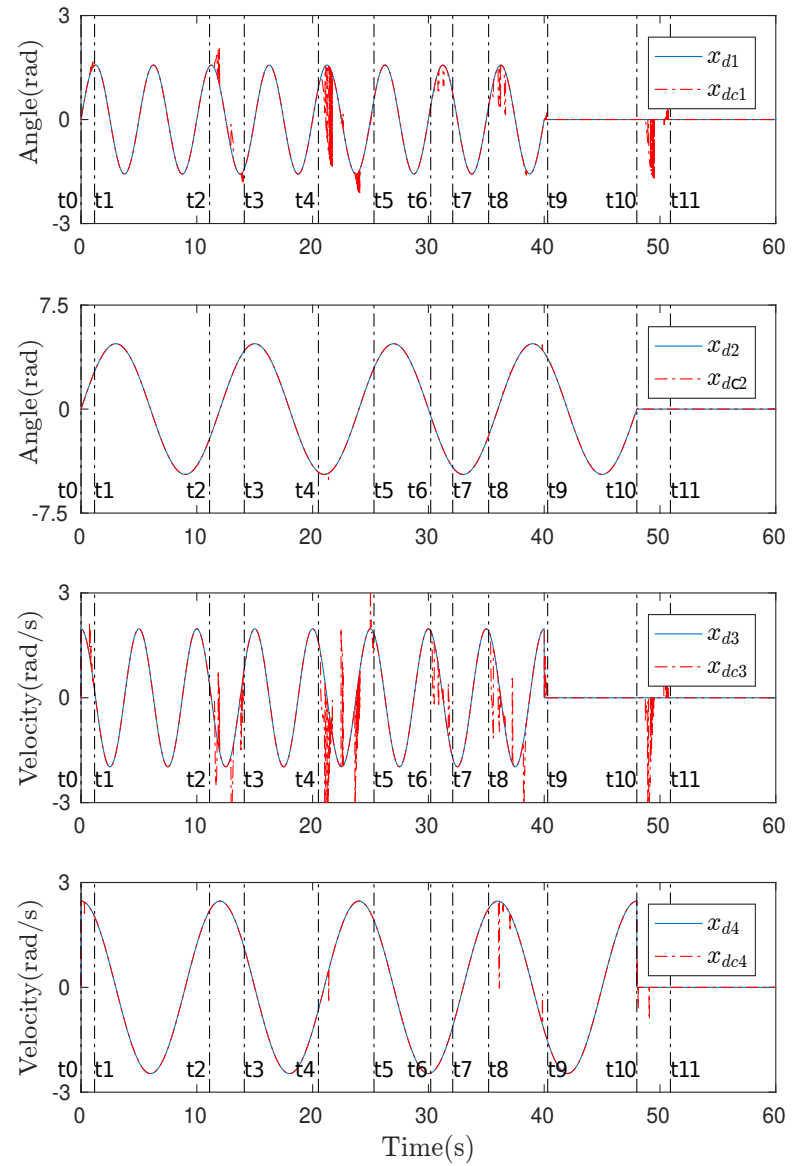

Fig. 7: Desired trajectory and modified desired trajectory

(ii) The parameters $f_{M}, f_{m}$ and $\gamma$ are selected as $f_{M}=$ $20, f_{m}=360, \gamma=100$. The feasible solution for LMI group (29),(41) and (42) is solved as

$$
H=\left[\begin{array}{cc}
-0.0115 & 0 \\
0 & -0.0115
\end{array}\right] .
$$

and $\eta=6.6282 \times 10^{-11}$.

(iii) The constant $\vartheta$ in (30) is chosen as $\vartheta=0.01$. The parameter matrix $G$ in the switching function $\hat{s}$ in (24) is 
chosen as $G=I_{2 n}$. The gain $K$ is chosen as

$$
K=\left[\begin{array}{cccc}
-50 & 0 & 0 & 0 \\
0 & -50 & 0 & 0 \\
-630 & 0 & -313 / 5 & 0 \\
0 & -4410 & 0 & -143
\end{array}\right] .
$$

The reference joint trajectory of the robot is chosen as

$$
\left\{\begin{array}{l}
x_{d, 1}=1.5 \sin \left(\frac{\pi}{5} t\right) \\
x_{d, 2}=5 \sin \left(\frac{\pi}{12} t\right)
\end{array}\right.
$$

The commanded torques on the robot are exerted from $t_{0}$. As shown in Tab. II, at the time $t_{2}$ and $t_{8}$, the robot is pulled by a rope tied on the endeffector, while at $t_{4}, t_{6}$ and $t_{10}$, the robot is hit by a ball. The safety bounds of the two manipulators are chosen as $\tau_{s, 1}=0.9 \mathrm{Nm}$ and $\tau_{s, 2}=1.9 \mathrm{Nm}$. The parameters in the saturation function (35) are selected as $\delta_{1}=2, k_{1}=2$, $k_{s, 1}=1, \delta_{2}=4, k_{2}=1.1$ and $k_{s, 2}=1$.

In order to make a comparison of our SMO results with the 'real' value in simulation, we calculate the 'real' state $\dot{q}$, $\ddot{q}$ and $\tau_{d}$ as below. The joint velocity $\dot{q}$ is obtained through a derivative filter $F_{1}(s)=\frac{s}{0.001 s+1}$ from $q$. The disturbance $\tau_{d}$ is calculated according to $\tau_{d}=\tau-u$, where the input torque $\tau$ is $\tau=M \ddot{q}+C(q, \dot{q}) \dot{q}+F(\dot{q})$. The acceleration $\ddot{q}$ is also obtained through a derivative filter $F_{2}(s)=\frac{s}{0.02 s+1}$ from $\dot{q}$.

The angular disturbance $\phi_{p, i}$ between obstacle and $i$-th link, for $i=1,2$, is assumed to be positive. From Fig. 3 it indicates that the full system state $\hat{x}$ have been correctly estimated. In Fig. 4, the reconstructed disturbance $\hat{\tau}_{d}$ roughly consists with the 'real' disturbance $\tau_{d}$. In Fig. 5, we introduce a flag signal indicating whether the estimated torque $\hat{\tau}_{d, i}$ on each joint exceeds the safety threshold $\tau_{s, i}$, with ' 1 ' for yes and ' 0 ' for no. It can be seen that in the first time interval, $t_{0}-t_{1}$, the threshold is exceeded due to the existence of static friction. During other intervals, $\left[t_{2}, t_{3}\right],\left[t_{4}, t_{5}\right],\left[t_{6}, t_{7}\right]$, $\left[t_{8}, t_{9}\right]$ and $\left[t_{10}, t_{11}\right]$, the contacts with the rope and the ball are successfully detected as shown in Fig. 5. During the time intervals when disturbance is strong, the control input is bounded to protect the robot as shown in Fig. 6; and the new compensated reference trajectory is calculated as shown in Fig. 7. While in other time intervals when the disturbance is weak, the desired trajectory is tracked by the manipulator.

\section{CONCLUSion}

A protective control framework based on state and disturbance estimation of robot manipulators using sliding mode method have been studied in this paper. The SMO is utilized to estimate the disturbance and the SMC is used to implement the protective reaction strategies according to different cases. A model transformation has been used to augment the nonlinear affine control system into a descriptor system, based on which the SMO is designed. By the estimated state and reconstructed torque disturbance, the SMC and protective strategies are proposed to follow the trajectory in weak disturbance and protect the robot manipulator in strong disturbance respectively. A 2-DOF manipulator experiment is conducted to examine the validity of our design schemes in this paper.

\section{APPENDIX I}

Following the ideas in [21], [27], [28], an LMI approach is introduced. The constraint (16) can be written as

$$
\text { Trace }\left[\left((H \bar{C})^{T}-\bar{B}_{f}^{T} \bar{E}^{-T} \bar{P}^{T}\right)^{T}\left((H \bar{C})^{T}-\bar{B}_{f}^{T} \bar{E}^{-T} \bar{P}^{T}\right)\right]=0 \text {. }
$$

Thus there exists a parameter $\eta>0$, such that

$$
\left((H \bar{C})^{T}-\bar{B}_{f}^{T} \bar{E}^{-T} \bar{P}^{T}\right)^{T}\left((H \bar{C})^{T}-\bar{B}_{f}^{T} \bar{E}^{-T} \bar{P}^{T}\right) \leq \eta I,
$$

where the parameter $\eta$ is related to the optimization (41)

$$
\min \eta \text {. }
$$

By Schur-complement, (40) is rebuilt as

$$
\left[\begin{array}{cc}
-\eta I & \left((H \bar{C})^{T}-\bar{B}_{f}^{T} \bar{E}^{-T} \bar{P}^{T}\right)^{T} \\
* & -I
\end{array}\right] \leq 0 .
$$

Thus, the bounded constraint (16) is solved by using the LMI equations (41) and (42) together.

\section{APPENDIX II}

For the given system (11), there exists a parameter $\kappa$ such that

$$
\operatorname{Re}\left[\lambda_{i}\left(-\left(\kappa I+\bar{E}^{-1} \bar{A}\right)\right)\right]<0, \forall i \in\{1,2, \ldots, 2 n\} .
$$

Given that $\left[-\left(\kappa I+\bar{E}^{-1} \bar{A}\right), \bar{C}\right]$ is observable, there exists a positive definite matrix $\bar{Q}$ such that

$$
-\bar{C}^{T} \bar{C}=\bar{Q}\left[\kappa I+\bar{E}^{-1}(\bar{A}-\bar{L} \bar{C})\right]+\left[\kappa I+\bar{E}^{-1}(\bar{A}-\bar{L} \bar{C})\right]^{T} \bar{Q},
$$

subject to

$$
\operatorname{Re}\left[\lambda_{i}\left(\bar{E}^{-1}(\bar{A}-\bar{L} \bar{C})\right)\right]<-\kappa, \forall i \in\{1,2, \ldots, \bar{n}\},
$$

where the gain matrix $\bar{L}$ defined as

$$
\bar{L}=\bar{E} \bar{Q}^{-1} \bar{C}^{T}
$$

and $\bar{E}^{-1}(\bar{A}-\bar{L} \bar{C})$ is Hurwitz.

\section{APPENDIX III}

For the error dynamics in (13) a Lyapunov function is defined as

$$
V(t)=\bar{e}^{T}(t) \bar{P} \bar{e}(t) .
$$

Thus we have

$$
\begin{aligned}
\dot{V}(t)= & 2 \bar{e}^{T}(t) \bar{P} \dot{\bar{e}}(t) \\
= & 2 \bar{e}^{T}(t) \bar{P} \bar{E}^{-1}\left[(\bar{A}-\bar{L} \bar{C}) \bar{e}(t)+\bar{B} e_{g}(\hat{x}, x, u, t)\right. \\
& \left.-\bar{L}_{f} u_{s}(t)+\bar{B}_{f} \bar{f}(t)\right],
\end{aligned}
$$

Subtituting (14) into (15) we have [27], [28]

$$
\begin{aligned}
& 2 \bar{e}^{T}(t) \bar{P} \bar{E}^{-1}\left[-\bar{L}_{f} u_{s}(t)+\bar{B}_{f} \bar{f}(t)\right] \\
= & -2\left(f_{M}+\lambda_{\max }\left(\Phi^{-1}\right) f_{m}+\zeta\right)\left|s^{T}(t)\right|+2 s^{T}(t) \bar{f}(t) \\
\leq & -2\left(f_{M}+\lambda_{\max }\left(\Phi^{-1}\right) f_{m}+\zeta\right)\left|s^{T}(t)\right|+2\left|s^{T}(t)\right||\bar{f}(t)| \\
\leq & 0 .
\end{aligned}
$$

According to $\bar{E}^{-1} \bar{B}=\bar{B}$ and Assumption 1 we have

$$
2 \bar{e}^{T}(t) \bar{P} \bar{E}^{-1} \bar{B} e_{g}(\hat{x}, u, t) \leq 2 \bar{e}^{T}(t) \gamma \bar{P}|\bar{B}| T_{e} \bar{e}(t)
$$


Subtituting (47) into (48) we have

$$
\dot{V}(t) \leq 2 \bar{e}^{T}(t)\left(\bar{P} \bar{E}^{-1}(\bar{A}-\bar{L} \bar{C})+\gamma \bar{P}|\bar{B}| T_{e}\right) \bar{e}(t) .
$$

If (17) is satisfied, then we have

$$
\dot{V}(t)<0
$$

and the error dynamics (13) is asymptotically stable.

\section{REFERENCES}

[1] D. Kulić and E. A. Croft, "Safe planning for human-robot interaction," Journal of Robotic Systems, vol. 22, no. 7, pp. 383-396, 2005.

[2] S. Haddadin, A. Albu-Schäffer, A. De Luca, and G. Hirzinger, "Collision detection and reaction: A contribution to safe physical human-robot interaction," in 2008 IEEE/RSJ International Conference on Intelligent Robots and Systems. IEEE, 2008, pp. 3356-3363.

[3] J. L. Novak and I. Feddema, "A capacitance-based proximity sensor for whole arm obstacle avoidance," in 1992 IEEE International Conference on Robotics and Automation. Proceedings. IEEE, 1992, pp. 1307-1314.

[4] D. M. Ebert and D. D. Henrich, "Safe human-robot-cooperation: Image-based collision detection for industrial robots," in 2002. IEEE/RSJ International Conference on Intelligent Robots and Systems, vol. 2. IEEE, 2002, pp. 1826-1831.

[5] D. Henrich and T. Gecks, "Multi-camera collision detection between known and unknown objects," in ICDSC 2008. 2008 Second ACM/IEEE International Conference on Distributed Smart Cameras. IEEE, 2008, pp. 1-10.

[6] V. Feliu and F. Ramos, "Strain gauge based control of single-link flexible very lightweight robots robust to payload changes," Mechatronics, vol. 15, no. 5, pp. 547-571, 2005.

[7] V. J. Lumelsky and E. Cheung, "Real-time collision avoidance in teleoperated whole-sensitive robot arm manipulators," IEEE Transactions on Systems, Man, and Cybernetics, vol. 23, no. 1, pp. 194-203, 1993.

[8] A. De Santis, B. Siciliano, A. De Luca, and A. Bicchi, "An atlas of physical human-robot interaction," Mechanism and Machine Theory, vol. 43 , no. 3, pp. 253-270, 2008.

[9] A. De Luca and R. Mattone, "Actuator failure detection and isolation using generalized momenta," in 2003. Proceedings of the 2003 IEEE International Conference on Robotics and Automation (ICRA), vol. 1. IEEE, 2003, pp. 634-639.

[10] A. De Luca, A. Albu-Schäffer, S. Haddadin, and G. Hirzinger, "Collision detection and safe reaction with the DLR-III lightweight manipulator arm," in 2006 IEEE/RSJ International Conference on Intelligent Robots and Systems. IEEE, 2006, pp. 1623-1630.

[11] A. De Luca and R. Mattone, "Sensorless robot collision detection and hybrid force/motion control," in Proceedings of the 2005 IEEE International Conference on Robotics and Automation. IEEE, 2005, pp. 999-1004.

[12] G. Tonietti, R. Schiavi, and A. Bicchi, "Design and control of a variable stiffness actuator for safe and fast physical human/robot interaction," in Proceedings of the 2005 IEEE International Conference on Robotics and Automation. IEEE, 2005, pp. 526-531.
[13] V. Utkin, J. Guldner, and J. Shi, Sliding mode control in electromechanical systems. CRC press, 2009, vol. 34.

[14] I. U. Vadim, "Survey paper variable structure systems with sliding modes," IEEE Transactions on Automatic Control, vol. 22, no. 2, pp. 212-222, 1977.

[15] C. P. Tan and C. Edwards, "Sliding mode observers for detection and reconstruction of sensor faults," Automatica, vol. 38, no. 10, pp. 18151821,2002

[16] M. Tursini, R. Petrella, and F. Parasiliti, "Adaptive sliding-mode observer for speed-sensorless control of induction motors," IEEE Transactions on Industry Applications, vol. 36, no. 5, pp. 1380-1387, 2000.

[17] B. Jiang, P. Shi, and Z. Mao, "Sliding mode observer-based fault estimation for nonlinear networked control systems," Circuits, Systems, and Signal Processing, vol. 30, no. 1, pp. 1-16, 2011.

[18] Z. Li, J. Yu, X. Xing, and H. Gao, "Robust output-feedback attitude control of a three-degree-of-freedom helicopter via sliding-mode observation technique," IET Control Theory \& Applications, vol. 9 , no. 11, pp. 1637-1643, 2015.

[19] B. Jiang, M. Staroswiecki, and V. Cocquempot, "Fault accommodation for nonlinear dynamic systems," IEEE Transactions on Automatic Control, vol. 51, no. 9, p. 1578, 2006.

[20] M. Chen and W.-H. Chen, "Sliding mode control for a class of uncertain nonlinear-system-based on disturbance observer," International Journal of Adaptive Control and Signal Processing, vol. 24, no. 1, pp. $51-64,2010$

[21] Y. Niu and D. W. Ho, "Robust observer design for Itô stochastic timedelay systems via sliding mode control," Systems \& Control Letters, vol. 55, no. 10, pp. 781-793, 2006.

[22] Z. Gao and H. Wang, "Descriptor observer approaches for multivariable systems with measurement noises and application in fault detection and diagnosis," Systems \& Control Letters, vol. 55, no. 4, pp. 304-313, 2006.

[23] A. De Luca and R. Mattone, "Sensorless robot collision detection and hybrid force/motion control," Proceedings - IEEE International Conference on Robotics and Automation, vol. 2005, no. April, pp. 999-1004, 2005.

[24] J. J. Craig, Introduction to robotics: mechanics and control. Pearson Prentice Hall Upper Saddle River, 2005, vol. 3.

[25] X.-G. Yan and C. Edwards, "Nonlinear robust fault reconstruction and estimation using a sliding mode observer," Automatica, vol. 43, no. 9, pp. 1605-1614, 2007.

[26] R. Rajamani, "Observers for Lipschitz nonlinear systems," IEEE transactions on Automatic Control, vol. 43, no. 3, pp. 397-401, 1998.

[27] M. Liu and P. Shi, "Sensor fault estimation and tolerant control for Itô stochastic systems with a descriptor sliding mode approach," Automatica, vol. 49, no. 5, pp. 1242-1250, 2013.

[28] H. Li, H. Gao, P. Shi, and X. Zhao, "Fault-tolerant control of markovian jump stochastic systems via the augmented sliding mode observer approach," Automatica, vol. 50, no. 7, pp. 1825-1834, 2014. 